\title{
The Dynamic Modeling of a Shape Memory Alloy
}

\author{
Ming Liu a , Daming Dong ${ }^{\text {b, }}$ Chun Dong ${ }^{c}$ \\ School of Beijing Jiaotong University, Beijing 100044, China. \\ a15121438@bjtu.edu.cn, b13291094@bjtu.edu.cn, cchdong@bjtu.edu.cn
}

Keywords: shape memory alloy, model identification, dynamic mode, dSPACE.

\begin{abstract}
This contribution providing a new test platform using FAUHABER linear motor and dSPACE is proposed based on the engineering application of shape memory alloy wire actuator. In this platform, a sinusoidal signal is input to the shape memory alloy wire to acquire the parameters. The dynamic model is identified by the system input-output and the MATLAB system identification toolbox. Compared with the mathematical model, the identified model made the description of shape memory alloy wire simple and effective, which lays the foundation for practical application.
\end{abstract}

\section{Introduction}

Shape Memory Alloy (SMA) is widely used in robotics, aerospace, medical treatment and other fields [1]. Its mechanical properties depend on the temperature and the applied stress. In microscopic the deformation of SMA is a change in martensite and austenite content. SMA is in the high-strength austenite phase during heating and becomes low-strength martensitic phase during cooling. SMA wire is easy to stretch under low temperature martensitic phase. With the temperature rising, SMA will return to the original shape. This feature can be used to construct the SMA actuators.

As a actuator, SMA wire has the advantages of a simple structure, large driving force, high energy density and so on. These advantages can simplify the actuator structure and reduce the costs. Many scholars made plentiful research on SMA actuator, such as F. Schiedesk [2], D. Js [3] and Dong Erbao [4]. The research direction mainly focuses on SMA modeling, control method and response frequency. There are many factors that affect the dynamic performance of SMA wire actuator, such as the geometric size, the phase transition temperature (Mf, Ms, As Af) [1], the electric heating method, the mechanical load and the ambient temperature [5] and so on. In the field of engineering applications, these factors make the SMA actuator mechanism modeling [2,6,7] process complicated.

The experimental platform uses FAULHABER precision linear motor as the stress and position sensor in the fan cooling conditions. Based on the input-output model identification method [3], The system identification toolbox of MATLAB is used to obtain the model which can meet the requirements and is easy to use. There are Hammerstein-Wiener model [2], Process model [8] and ARX model [9] and so on in the system identification toolbox. Compared with the mechanism model, the identification model can not reflect the mechanism of SMA, but it can correctly reflect the corresponding relationship between the physical quantity of SMA, and its flexibility makes the engineering application of SMA easy.

\section{A New Experimental Platform}

\subsection{The Structure of Platform.}

The experiment platform used to study the dynamic behavior of SMA actuators is shown in Fig. 1 and include the following sections:

1) SMA system: BMF150 SMA wire of Biometal, constant speed cooling fan, current source by precision power amplifier.

2) Sensor: LM1247-020-01 servo linear motor of FAULHABER.

3) dSPACE system: DS1005 PPC, DS2103, interface board. 


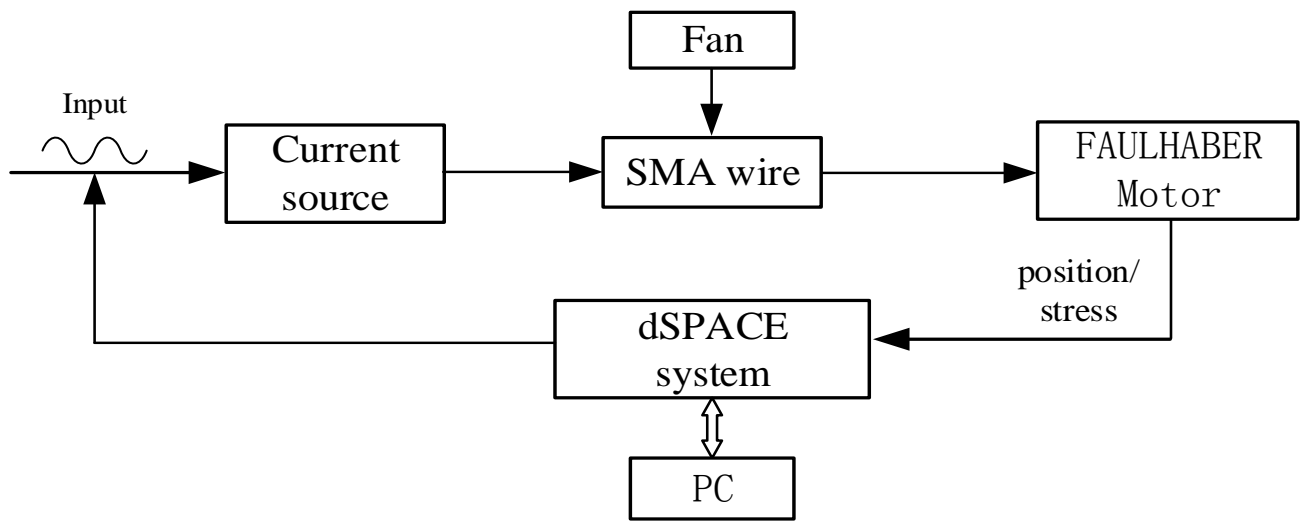

Fig. 1 Experimental platform for model identification

The length of the SMA wire used in the experiment was $425 \mathrm{~mm}$, the diameter is $0.15 \mathrm{~mm}$, and the standard driving force is $1.41 \mathrm{~N}$. In dSPACE system, DS1005 PPC is a hardware core, and its basic frequency is $1 \mathrm{GHz}$. The DS2103 is a fast D/A board with a sampling time of $10 \mu \mathrm{s}$.

\subsection{Design of Current Source.}

Amplifier to build the current source as shown in Fig. 2.

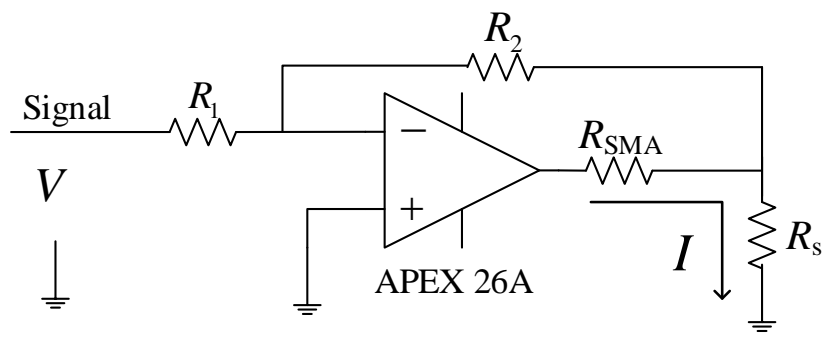

Fig. 2 Current source schematic

where $R_{\mathrm{S}}$ is the sampling power resistance, $R_{\mathrm{SMA}}$ is the shape memory alloy wire resistance, $R_{1}$ and $R_{2}$ are the proportional amplification resistance. In the figure, $V$ is the input signal voltage of DS2103 and $I$ is the current signal through SMA wire. The relationship between $V$ and $I$ is given by formula:

$$
I=-V \times \frac{R_{2}}{R_{1}} \times \frac{1}{R_{S}}
$$

\subsection{FAULHABER Linear Motor as a Sensor.}

Due to the special geometry of the SMA wire and the particularity of the installation method, the stress and displacement sensor have a great influence on the SMA wire dynamic data measurement. The traditional stress and displacement sensor should be composed of two parts, due to the size and the installation error of the multi-sensor, the experiment selected FAULHABER linear motor as a sensor. The motor's QUICKSHAFT ${ }^{\circledR}$ technology provides excellent dynamic characteristics, high force index and other advantage. The theoretical repeated positioning accuracy can reach $0.04 \mathrm{~mm}$, the linear relationship between the force of the motor shaft and the motor current is $\mathrm{Kf}=6.43 \mathrm{~N} / \mathrm{A}$, the current resolution is up to $10 \mathrm{~mA}$. Compared to use of weights to provide a constant load, the motor can avoid the error of dynamic process.

\section{Identification of Dynamics Model}

\subsection{Simulink Program.}

Before the model identification, the Simulink model was established as shown in Fig. 3. The model is automatically downloaded to the dSPACE by code generation to complete the signal reference and data acquisition. The input signal is a sine wave at different frequencies, and the resulting output data is processed to obtain a frequency response curve. The motor acquires the displacement data in the constant force mode and establishes the position model. In the same way, the stress data is acquired in the constant position mode, and the current or voltage can be viewed in real time. 


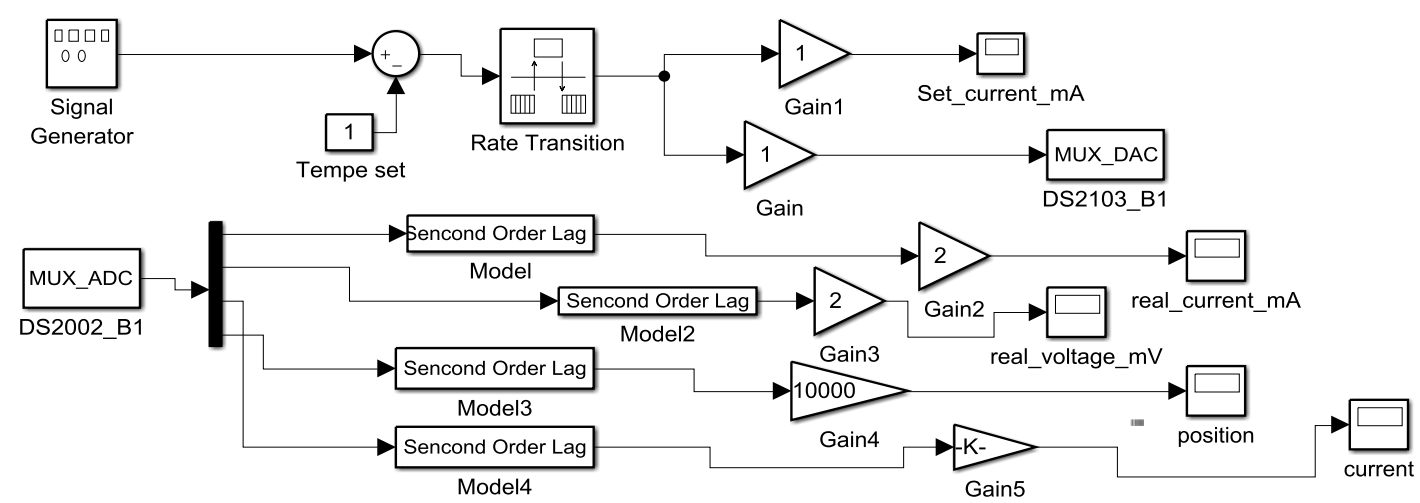

Fig. 3 Simulink model in DS1105

\subsection{Data Processing and Model Identification.}

The experiment uses the Control Desk to save the input and output data as a *.mat file, and the data was preprocessed to obtain the frequency response curve. Then select the parameters of the transfer function to be determine the poles and zeros.

Model identification process shown in Fig. 4. In the process of model identification, the input data should be sufficient so that the model can accurately describe the behavior of the system.

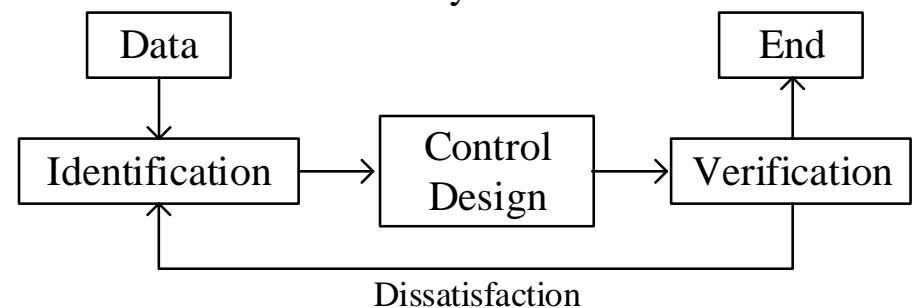

Fig. 4 Iterative process of model identification

After the comparative analysis, this paper selects two transfer functions to describe force model and position model of SMA wire. In order to verify the validity of the model, a set of contrast experiments were designed. The same set of signals were added to the SMA wire. Force model select $0.05,0.2 \mathrm{~Hz}$ of the sinusoidal signal as the input, the position model selects $0.05,0.2 \mathrm{~Hz}$ sinusoidal signal as the input, and the actual response of the SMA wire was measured. Using the system identification toolbox of MATLAB, the experiment got the following model:

a) Stress Model: $G(s)=\frac{9.237 s+438.9}{s^{2}+16.94+25.24}$

b) Position Model: $G(s)=\frac{20.04}{s^{2}+5.595 s+6.098}$

These two models are second-order transfer function, and the system response with degree of $91.61 \%$ and $93.27 \%$ respectively.

In order to verify the effect of two models, the model was imported into Simulink, and the sinusoidal signals was input to the model to capture the response and compared with the actual data. The results as shown in Fig. 5.

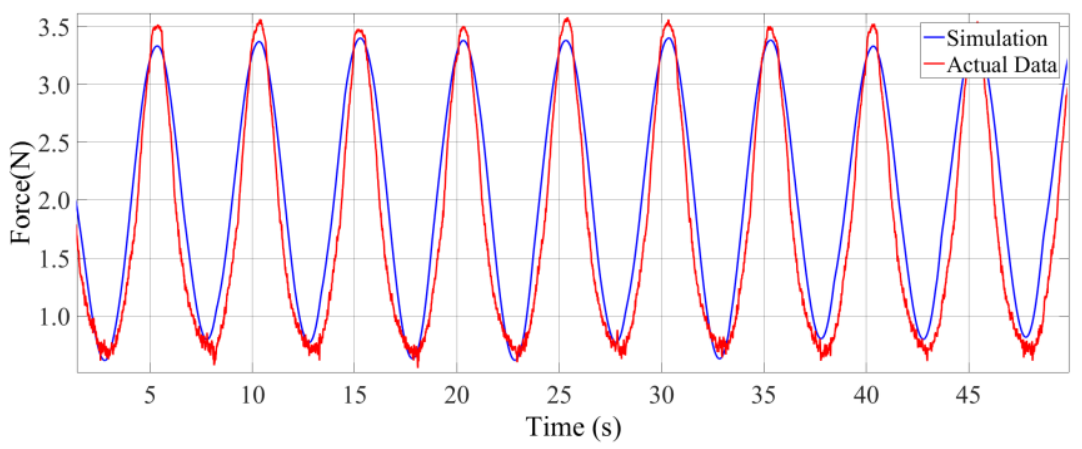

a) Stress model of $0.2 \mathrm{~Hz}$ sine signal 


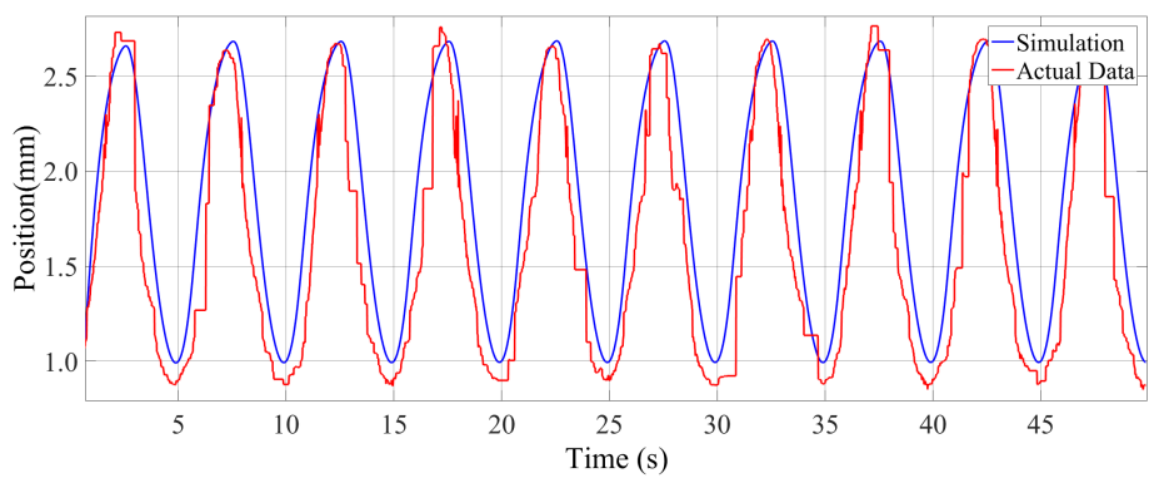

b) Position model of $0.2 \mathrm{~Hz}$ sine signal

Fig. 5 System response and actual data

As can be seen from the figure above, the model fit well in amplitude and phase within $0.2 \mathrm{~Hz}$. The presence of non-linearity makes the waveform looks not so "Sine". This paper adopts identified linear model to describe the relationship between the input power and the outputs(stress and position) of SMA.

\section{Conclusion}

This article utilize a new measurement method and second order transfer function model of SMA that represent force and position response is obtained by system identification. The experiment has been made to verify the dynamic performance of the model and the rationality of using the linear motor as the constant load. The identified model can reflect the dynamic response of SMA wire as force and position model, which simplify the control of SMA wire in engineering application. The result help making the application of SMA wire actuator more extensive, which has great significance.

\section{References}

[1]. Xu Zuyao, Jiang Bohong. Shape memory materials [M]. Shanghai: Shanghai Jiao Tong University press, 2000:2-10.

[2]. Schiedeck F, Mojrzisch S. Frequency-domain control design for shape memory alloy actuators[J]. Sensors \& Actuators A: Physical, 2011, 169(1): 133-140.

[3]. Js D, Sn S. Differential resistance feedback control of a self-sensing shape memory alloy actuated system[J]. Isa Transations, 2014, 53(2): 289.

[4]. Dong Erbao, Xu Min, Li Yongxin, Yang Jie. The new electric heating method of shape memory alloy wire actuator and its modeling and experimental research of [J]. China mechanical engineering, 2010 (23): 2857-2861.

[5]. Meier H, Oelschlaeger L. Numerical thermomechanical medeling of shape memory wires[J]. Materials Science and Engineering, 2004, 378(1-2): 484-489.

[6]. Dutta S M, Ghorbel F H. Differential Hysteresis Modeling of a Shape Memory Alloy Wire Actuator[J]. IEEE/ASME Transactions on Mechatronics, 2005, 10(2), 189-197.

[7]. Dutta S M, Ghorbel F H, Dabney J B. Modeling and Control of a Shape Memory Alloy Actuator. IEEE International Symposium on Intelligent Control, 2005: 1007-1012.

[8]. Villoslada A, Flores A, Blanco D, Moreno L. High-dispalcement flexible Shape Memory Alloy actuator for soft wearable robots[J]. Robotics and Autonomous Systems, 2015, 73: 91-101.

[9]. Cosma I A, Măties V, Lâpusan C, Mîndru R C. Modeling of Shape Memory Alloy Actuators Using Matlab and dSpace Platform[J]. Solid State Phenomena, 2010, 166-167:149-154. 